\title{
PERCEPÇÕES DA ENFERMAGEM ACERCA DA COMUNICAÇÃO TERAPÊUTICA EM SAÚDE MENTAL: UMA REVISÃO DE LITERATURA
}

\author{
NURSING PERCEPTIONS ABOUT THERAPEUTIC \\ COMMUNICATION IN MENTAL HEALTH: A LITERATURE REVIEW
}

\author{
Gabriela Portela ${ }^{1}$ \\ Helena Vitória Silva Pinheiro ${ }^{2}$ \\ Júlia Maria Vasconcelos Dias Moura ${ }^{3}$ \\ Marília Juliane Pedrosa Gurgel ${ }^{4}$ \\ Nathalia de Oliveira Burgo ${ }^{5}$ \\ Paola Frassinetti de Oliveira Correia ${ }^{6}$
}

RESUMO: A comunicação pode ser compreendida como um meio através do qual os indivíduos relacionam-se entre si e com o ambiente em que estão inseridos. Mediante os vínculos que devem ser estabelecidos entre profissionalpaciente, a comunicação se encorpa e torna-se terapêutica. Possivelmente, o campo da saúde mental é o mais enfático quanto à necessidade da comunicação terapêutica, pois é o que mais evidencia alterações e interrupções no processo de comunicar. Diante disso, este estudo tem o intuito de realizar uma revisão de literatura sobre as percepções da enfermagem acerca da comunicação terapêutica em saúde mental, como norte o questionamento: "Qual a percepção da enfermagem acerca da comunicação terapêutica utilizada com portadores de transtornos mentais?". Na busca, ao todo foram encontrados 464 artigos, 445 da Biblioteca Virtual em Saúde (BVS) e 19 da biblioteca virtual SciELO, conduzido a partir das recomendações das diretrizes do PRISMA. Sendo 4 artigos

\footnotetext{
${ }^{1}$ Discente do $9^{\circ}$ módulo do curso de Enfermagem da Faculdade de Enfermagem Nossa Senhora das Graças da Universidade de Pernambuco (UPE).

${ }^{2}$ Discente do $9^{\circ}$ módulo do curso de Enfermagem da Faculdade de Enfermagem Nossa Senhora das Graças da Universidade de Pernambuco (UPE).

${ }^{3}$ Discente do $9^{\circ}$ módulo do curso de Enfermagem da Faculdade de Enfermagem Nossa Senhora das Graças da Universidade de Pernambuco (UPE).

${ }^{4}$ Discente do $9^{\circ}$ módulo do curso de Enfermagem da Faculdade de Enfermagem Nossa Senhora das Graças da Universidade de Pernambuco (UPE).

${ }^{5}$ Discente do $9^{\circ}$ módulo do curso de Enfermagem da Faculdade de Enfermagem Nossa Senhora das Graças da Universidade de Pernambuco (UPE).

${ }^{6}$ Discente do $9^{\circ}$ módulo do curso de Enfermagem da Faculdade de Enfermagem Nossa Senhora das Graças da Universidade de Pernambuco (UPE).
} 
selecionados para a revisão sistemática. A partir da análise dos artigos, foi possível constatar que os discentes percebem que a comunicação terapêutica envolve não só a comunicação verbal, mas também os sentidos. Já os profissionais em sua maioria percebem a comunicação terapêutica como instrumento que aumenta a eficácia da relação terapêutica, atende a individualidade das pessoas, identifica e responde às verdadeiras necessidades de saúde das pessoas. Sendo assim, a comunicação terapêutica é ferramenta para efetivar relacionamentos terapêuticos, bem como auxiliar na oferta de cuidados em saúde e promover qualidade de vida aos pacientes psiquiátricos.

Palavras chave: Enfermagem, Saúde Mental, Comunicação em Saúde.

ABSTRACT: Communication can be understood as a medium through which individuals relate to each other and to the environment in which they are inserted. Through the bonds that must be established between professional-patient, communication becomes stronger and becomes therapeutic. Possibly, the field of mental health is the most emphatic regarding the need for therapeutic communication, as it's the one that most evidences, changes and interruptions in the process of communicating. Therefore, this study aims to conduct a literature review on the perceptions of nursing about therapeutic communications in mental health as the question: "What is the perception of nursing about the therapeutic communication used with patients with mental disorders?". In the search, 464 articles were found, 445 from the Virtual Health Library and 19 from the SciELO virtual library, based on recommendations from the PRISMA guidelines. Four Articles were selected for the systematic review. From the analysis of the articles, it was found that the students perceive that therapeutic communication involves not only verbal communication, but also the senses and body language. Most professionals, on the other side, perceive therapeutic communication as an instrument that increases the effectiveness of the therapeutic relationship, meets people's individuality, identifies and responds to people's real health needs. In conclusion, therapeutic communication is a tool for establishing therapeutic relationships, as well as assisting in the provision of health care and promoting therapeutic relationships, as well as assisting in the provision of health care and promotion quality of life for psychiatric patients.

Descriptors: Nursing, Mental Health, Health Communication. 


\section{INTRODUÇÃO}

O termo comunicar no latim, communicare, significa colocar em comum. Assim sendo, a comunicação pode ser compreendida como um instrumento através do qual os indivíduos relacionam-se entre si e com o ambiente em que estão inseridos, de maneira a produzir e reproduzir significação à símbolos através da mensagem verbal e não-verbal (SILVA; BARROS, 2015). Nesse sentido, a comunicação funciona como centro do processo do pensamento humano, mediatizando o pensar de um sujeito a outro (BRANCO; CIRINO, 2016). Ao entender a comunicação como ponto essencial na interação entre indivíduos, torna-se consenso a compreensão acerca da sua importância como instrumento de intervenção na área da saúde. É através do ato de comunicar que o profissional da enfermagem se insere no dia a dia do usuário, seja para orientar, informar, escutar, apoiar ou entender suas necessidades e demandas. A enfermagem é uma profissão que lida com o cuidar, assim pode perceber 0 paciente, a família, as demandas, as necessidades, em prol da sua formação, mas, principalmente, da visão holística quanto ao cuidado que lhe concerne. Portanto, a comunicação também configura-se como parte essencial da assistência prestada pela equipe de enfermagem (COELHO, 2015; CENCl, 2015; SALVIANO et al, 2016).

Mediante os vínculos de confiança que devem ser estabelecidos entre profissional-paciente, a comunicação se encorpa e torna-se terapêutica. A partir de fundamentos teóricos e de habilidades interpessoais adquiridas, o enfermeiro passa a agir planejadamente e individualmente na assistência do usuário (JALLES; SANTOS; REINALDO, 2017; PEREIRA et al, 2019).

O campo da saúde mental é, possivelmente, o mais enfático quanto à necessidade da comunicação terapêutica pois é o que mais evidencia alterações e interrupções no processo de comunicar. Esse campo específico, e ainda menosprezado, era visto apenas a partir do modelo clínico-biológico, o usuário 
era somente um doente em crise. Com o advento da Reforma Psiquiátrica no Brasil, emergiram discussões acerca da concepção dos pacientes com transtornos mentais enquanto seres humanos, bem como as formas de tratamento (JALLES; SANTOS; REINALDO, 2017; RADKE; CECCIM, 2018).

O sofrimento psíquico passou a ser entendido como um processo subjetivo, social e cultural que decorre de alterações psíquicas vivenciadas como também de prejuízos nas relações pessoais, na vida social e afetiva (PAIVA, 2018). Nessa perspectiva, é somente através da comunicação que as perturbações, angústias e limitações do usuário serão percebidas.

Através da análise das literaturas nota-se o valor da comunicação como forma terapêutica na construção e no andamento da relação da enfermagem, como profissional ou discente, para com o portador de transtornos mentais. Considerando a comunicação como uma ferramenta que viabiliza o contato e estabelecimento de vínculo entre usuário-enfermeiro, é fundamental não só compreendê-la, como também saber como acontece a comunicação em saúde utilizada por profissionais de enfermagem com portadores de transtornos mentais, tendo em vista a grande lacuna existente acerca da temática.

Diante disso, este estudo tem o intuito de realizar uma revisão sistemática da literatura sobre as percepções da enfermagem acerca da comunicação terapêutica em saúde mental, a partir da seguinte pergunta condutora: "Qual a percepção da enfermagem acerca da comunicação terapêutica utilizada com portadores de transtornos mentais?"

\section{MÉTODO}

A revisão de literatura foi realizada com base na pergunta condutora: "Qual a percepção da enfermagem acerca da comunicação terapêutica utilizada com portadores de transtornos mentais?". O estudo foi conduzido a partir das recomendações das diretrizes do protocolo PRISMA - Principais Itens para Relatar Revisões sistemáticas e Meta-análises (GALVÃO; PANSANI; HARRAD, 
2015). No que se refere à seleção dos artigos, foram consultadas as bases de dados da Biblioteca Virtual em Saúde (BVS) e SciELO (Scientific Electronic Library Online), com a seguinte estratégia de busca: enfermagem and saúde mental and comunicação em saúde.

Os critérios de inclusão para o estudo foram: publicações que abordem a temática da comunicação em saúde entre profissionais e estudantes de enfermagem com portadores de doenças psíquicas, no período de publicação entre os anos de 2014 a 2018 e textos disponíveis na íntegra. A busca de referências ocorreu de outubro a novembro de 2018 e limitou-se a publicações nos idiomas português e inglês.

A partir disso, todos os resumos dos artigos encontrados foram lidos, sendo excluídos os que não correspondiam aos critérios de inclusão prédefinidos e aqueles que causaram questionamentos sobre sua elegibilidade, foram consultados na íntegra. Posteriormente, os artigos resultantes foram avaliados na versão completa para confirmar sua inclusão no estudo e os que estavam presentes em duas bases de dados foram contabilizados apenas uma vez, excluindo as duplicatas.

A análise das publicações encontradas através da busca foi realizada de forma descritiva, em duas etapas. A primeira delas incluiu examinar as variáveis: ano, autoria, local, tipo e delineamento do estudo, população-alvo, método de avaliação do desfecho quanto à elaboração da pergunta norteadora. A etapa seguinte consistiu em verificar a prevalência do desfecho analisado, associado a seus fatores causais.

\section{RESULTADOS}

$\mathrm{Na}$ busca, como pode ser observado na figura 1, foram encontrados 464 artigos, 445 da Biblioteca Virtual em Saúde e 19 da biblioteca virtual SciELO. Em relação às bases de dados onde estavam disponíveis apresentavam-se: 396 na Medline, 24 na LILACS, 23 na BDENF, 2 na Index - Psicologia, e 19 disponíveis 
na SciELO. De início, foram eliminados 11 artigos por duplicação, e após análise dos títulos eresumos foram eliminados 426 .

Dos 38 artigos elegíveis, foram excluídos 34 artigos pelos seguintes critérios de exclusão: 26 não respondiam a pergunta norteadora, sendo ela: "Qual a percepção da enfermagem acerca da comunicação terapêutica utilizada com portadores de transtornos mentais?"; 4 foram excluídos por impossibilidade de acesso aos textos completos nas bases; e 4 foram eliminados por não serem teses, que não caberiam dentro da respectiva revisão.

FIGURA 1. Fluxograma de identificação e seleção dos artigos para revisão sistemática sobre percepção da enfermagem em relação a comunicação terapêutica utilizada em saúde mental, 2014 a 2018.

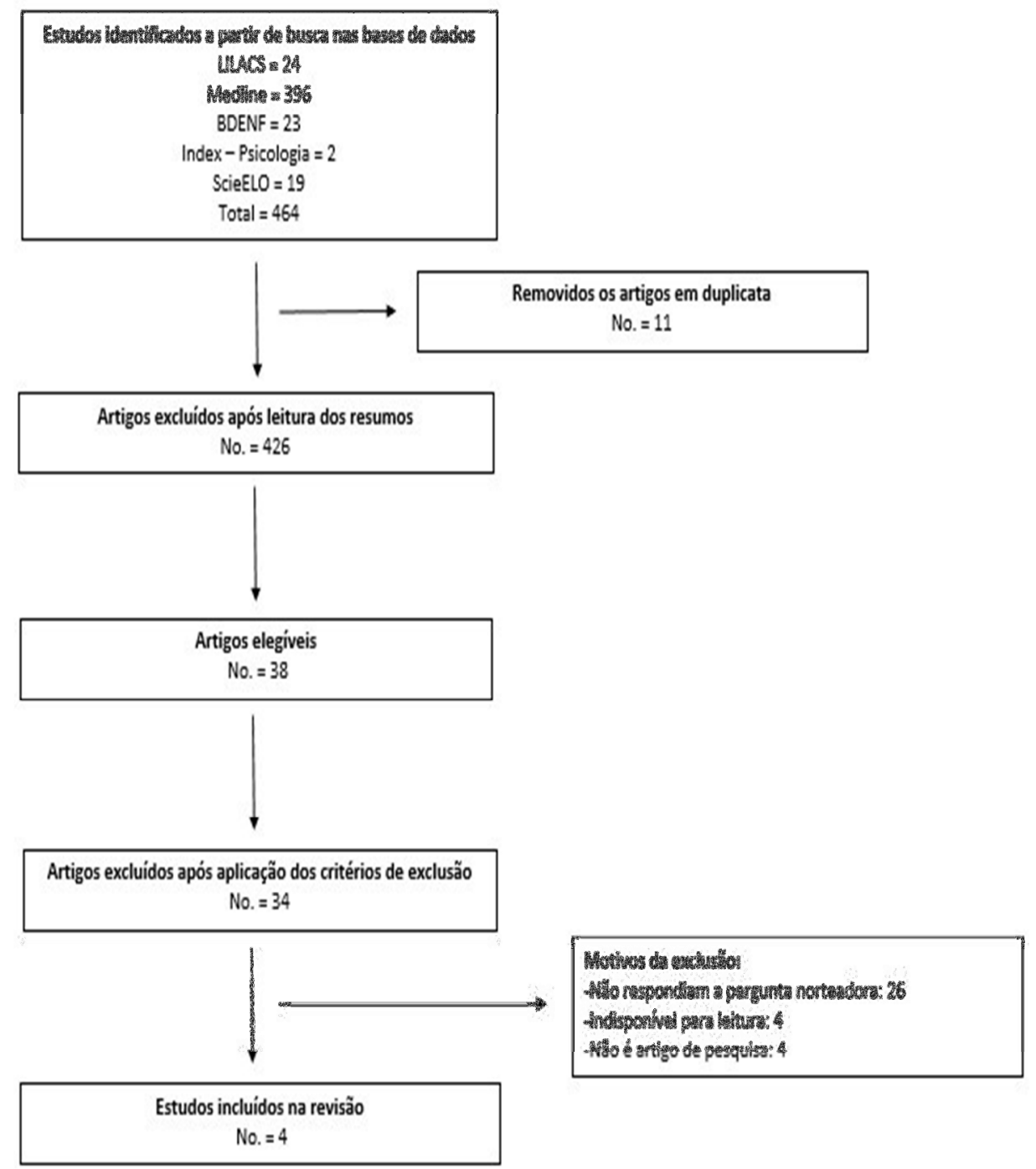


No fim, 4 artigos foram selecionados para a realização da revisão de literatura. Todos os 4 artigos trazem uma pesquisa do tipo descritiva e exploratória, sendo 3 de caráter qualitativo e 1 de caráter quantitativo. Em relação à amostra dos artigos, observa-se que três deles utilizaram a mesma amostra, com 23 participantes, sendo eles graduandos(as) do sétimo período do curso de graduação em Enfermagem, e 1 artigo contou com 448 enfermeiros(as).

Tomando como base o ano de publicação, o mais antigo foi de 2014, o qual se referia à pesquisa com os(as) enfermeiros(as). Os demais artigos foram um de 2015 e os outros dois de 2017. Já os locais do estudo, o que referia-se aos enfermeiros(as) foi realizado em Portugal e os outros três aconteceram no Brasil, mais especificamente no estado do Rio de Janeiro. Para uma melhor visualização dos arquivos pode-se ter como base a tabela 1.

TABELA 1. Características dos estudos sobre percepção da enfermagem em relação a comunicação terapêutica utilizada em saúde mental segundo título, autor, ano, base de dados, tipo de estudo, amostra e tipo da amostra, 2014 a 2018.

\begin{tabular}{|c|c|c|c|c|c|c|c|}
\hline $\mathbf{N}$ & Título & Autor & $\begin{array}{c}\text { Ano de } \\
\text { publicação }\end{array}$ & $\begin{array}{c}\text { Base de } \\
\text { dados }\end{array}$ & $\begin{array}{l}\text { Tipo de } \\
\text { estudo }\end{array}$ & Amostra & Tipo da amostra \\
\hline 1 & $\begin{array}{l}\text { Comunicação } \\
\text { Terapêutica em } \\
\text { Enfermagem: } \\
\text { Como a } \\
\text { caracterizam os } \\
\text { enfermeiros }\end{array}$ & $\begin{array}{l}\text { Coelho; } \\
\text { Sequeira }\end{array}$ & 2014 & SciELO & $\begin{array}{l}\text { Quantitativo, } \\
\text { exploratório, } \\
\text { descritivo e } \\
\text { transversal }\end{array}$ & 448 & Enfermeiros \\
\hline 2 & $\begin{array}{c}\text { Como o } \\
\text { estudante de } \\
\text { enfermagem } \\
\text { percebe a } \\
\text { comunicação } \\
\text { com o paciente } \\
\text { em saúde } \\
\text { mental }\end{array}$ & $\begin{array}{l}\text { Azevedo; } \\
\text { Araújo; } \\
\text { Vidal }\end{array}$ & 2015 & LILACS & $\begin{array}{l}\text { Qualitativo, } \\
\text { descritivo e } \\
\text { exploratório }\end{array}$ & 23 & $\begin{array}{l}\text { Graduandos(as) } \\
\text { de Enfermagem }\end{array}$ \\
\hline
\end{tabular}




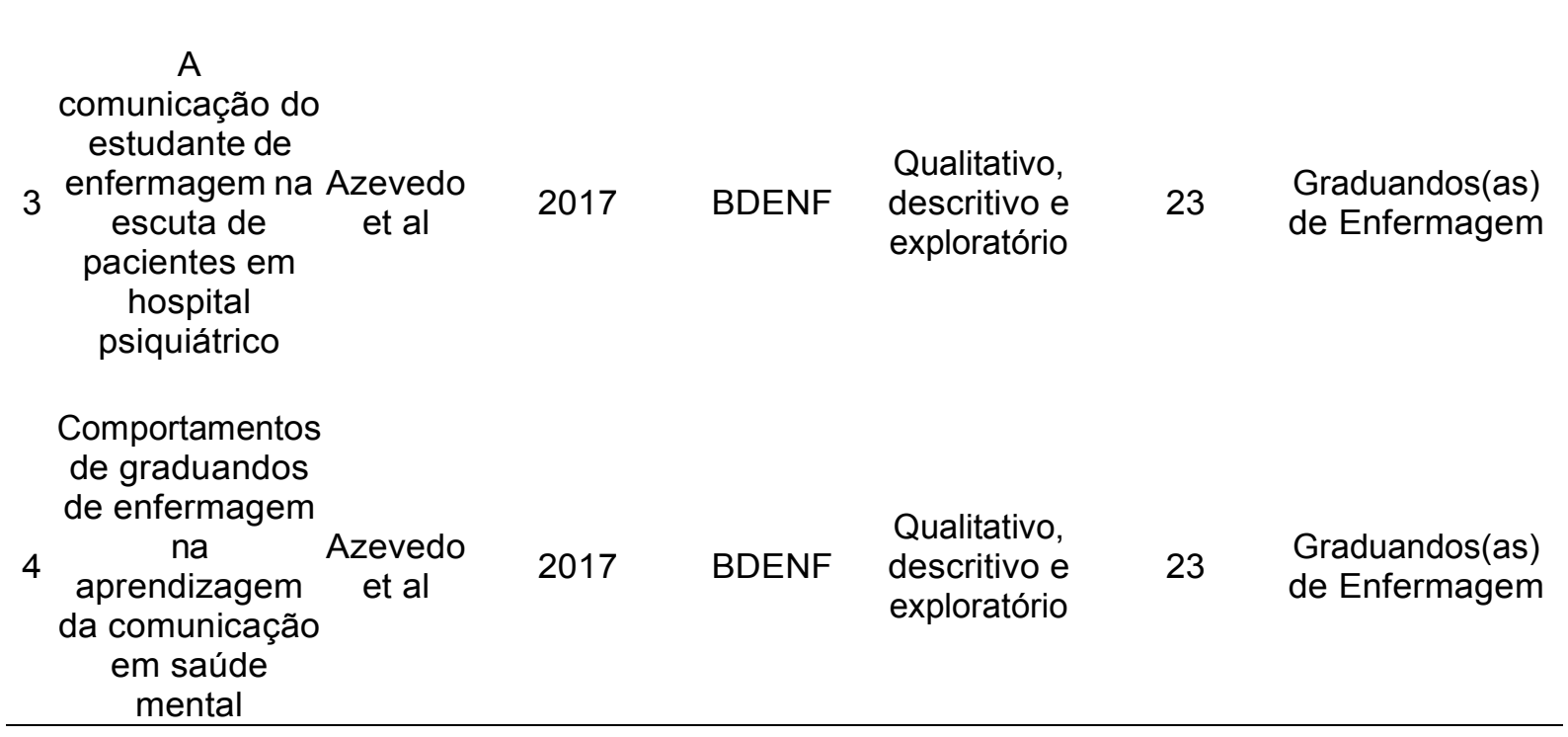

Três artigos investigaram os comportamentos e percepções na comunicação dos graduandos com os portadores de doenças psíquicas. Nestes estudos destacou-se, a partir dos depoimentos e experiências dos alunos, a utilização dos sentidos corporais como instrumento que pode vir a colaborar ou a interferir negativamente na interação estudante - paciente.

Concepção dos estudantes acerca de alguns sentidos:

Olfativo

"Mesmo que o odor exalado pelo paciente esteja muito forte, na psiquiatria temos que interagir e vamos sentar e conversar" (AZEVEDO et al, 2017b).

Visão

"A percepção visual contribui com a avaliação do estado de humor e do comportamento do paciente. A partir desta avaliação, podemos conduzir e estabelecer os cuidados necessários, avaliar determinados componentes da psicopatologia ou qualquer outra situação que pode nos conduzir e determinar metas, implementar ações e futuras intervenções" (AZEVEDO et al, 2017b).

Um dos estudos analisou com mais ênfase um dos sentidos: a audição. No qual, os estudantes perceberam a escuta como uma oportunidade de compreensão da comunicação verbal.

"Minha intenção foi procurar ouvir o paciente. As angústias (...) as preocupações, para poder ver o que se pode fazer por ele. Porque é muito fácil deixar o paciente ali calado, excluído, cabisbaixo. Agora, você ir lá, conversar 
com ele, ver o que está acontecendo, o que está passando na cabeça dele é outra coisa" (AZEVEDO et al, 2017a).

No que diz respeito, às percepções da comunicação entre profissionais e pessoas com sintomas psiquiátricos foi encontrado apenas um artigo. Através dele, observou-se que a maioria dos(as) enfermeiros(as) percebem a comunicação terapêutica como instrumento que aumenta a eficácia da relação terapêutica, atende a individualidade das pessoas, identifica e responde às verdadeiras necessidades de saúde das pessoas. Todavia, através do estudo foi possível identificar que uma parcela dos entrevistados não percebe a comunicação terapêutica como um ato intencional e com valor clínico autónomo (COELHO; SEQUEIRA, 2014).

TABELA 2. Características dos estudos sobre percepção da enfermagem em relação a comunicação terapêutica utilizada em saúde mental segundo título, autor, ano de publicação e desfechos, 2014 a 2018.

\begin{tabular}{|c|c|c|c|c|}
\hline $\mathbf{N}$ & Título & Autores & $\begin{array}{c}\text { Ano de } \\
\text { publicação }\end{array}$ & Desfechos \\
\hline 1 & $\begin{array}{l}\text { Comunicação } \\
\text { Terapêutica em } \\
\text { Enfermagem: } \\
\text { Como a } \\
\text { caracterizam os } \\
\text { enfermeiros }\end{array}$ & $\begin{array}{l}\text { Coelho; } \\
\text { Sequeira }\end{array}$ & 2014 & $\begin{array}{l}\text { Globalmente existe uma manifesta } \\
\text { concordância dos inquiridos em relação aos } \\
\text { aspectos caracterizadores da comunicação } \\
\text { terapêutica, salientando-se no entanto o fato de } \\
\text { esta concordância ser menor quando se refere } \\
\text { ao uso intencional e ao valor clínico autónomo, } \\
\text { fundamentais para o desenvolvimento da } \\
\text { dimensão autónoma dos cuidados. }\end{array}$ \\
\hline 2 & $\begin{array}{c}\text { Como o } \\
\text { estudante de } \\
\text { enfermagem } \\
\text { percebe a } \\
\text { comunicação com } \\
\text { o paciente em } \\
\text { saúde mental } \\
\end{array}$ & $\begin{array}{l}\text { Azevedo; } \\
\text { Araújo; } \\
\text { Vidal }\end{array}$ & 2015 & $\begin{array}{l}\text { Os sentidos corporais dos estudantes de } \\
\text { Enfermagem registram e expressam a } \\
\text { comunicação verbal e não verbal do paciente } \\
\text { com transtorno mental por meio da sensação, } \\
\text { do comportamento e das condições do corpo } \\
\text { que emanaram necessidades de cuidado. }\end{array}$ \\
\hline 3 & $\begin{array}{l}\text { A comunicação do } \\
\text { estudante de } \\
\text { enfermagem na } \\
\text { escuta de } \\
\text { pacientes em } \\
\text { hospital } \\
\text { psiquiátrico }\end{array}$ & $\begin{array}{l}\text { Azevedo et } \\
\quad \text { al }\end{array}$ & 2017 & $\begin{array}{l}\text { Nesta experiência, que marcou o processo de } \\
\text { ensino-aprendizagem na área da saúde mental, } \\
\text { a comunicação não verbal proxêmica fo } \\
\text { unanimemente referida pelos estudantes de } \\
\text { enfermagem, pela valorização da posição } \\
\text { pessoal-corporal mantida, rosto, pescoço e } \\
\text { ombros durante a escuta de pacientes em } \\
\text { sofrimento psíquico. O raciocínio clínico } \\
\text { apresentou-se como uma competência } \\
\text { importante à escuta, que, ao auxiliar o } \\
\text { estudante, favoreceu o reconhecimento das } \\
\text { desordens na fala e no pensamento, }\end{array}$ \\
\hline
\end{tabular}


melhorando a interação e a escuta.

Comportamen tos de graduandos de 4 enfermagem na Azevedo et aprendizagem da comunicação em saúde mental
Os sentidos corporais, utilizados como elemento norteador da produção artística dos graduandos, funcionaram como elementos fundamentais para a produção de conhecimento. O sentido visão permitiu o reconhecimento de si e do outro na interação. O sentido paladar definiu a forma

2017 de se relacionar no mundo, as ligações e experiências entre as pessoas e as coisas. $O$ sentido olfato permitiu detectar os cuidados ausentes na hospitalização. Houve transversalidade entre o sentido paladar e o sentido olfato, na utilização de figuras de linguagem.

\section{DISCUSSÃO}

A comunicação envolve compartilhar informações e compreender o outro, seja verbal ou não verbalmente, está intimamente ligada aos sentimentos e à vontade de interação, emitindo e recebendo mensagens para que haja compreensão de ambas as partes sobre o que se quer transmitir aos indivíduos (ANDRADE et al, 2015). A Comunicação Terapêutica em enfermagem é uma interação entre profissional-paciente, uma forma de aproximação necessária durante o processo do cuidar, pois transmite-lhes segurança e confiança (SILVA; BARROS, 2015).

Através da análise dos resultados referente aos artigos selecionados para a revisão, percebeu-se a necessidade de separar didaticamente a discussão a respeito da percepção da comunicação terapêutica realizada para a enfermagem em dois tópicos, de forma a entender como a temática perpassa desde os primórdios da graduação até o exercício da profissão. 


\section{Comunicação terapêutica entre enfermeiro e pacientes com transtornos mentais}

No artigo "Comunicação Terapêutica em Enfermagem: Como a caracterizam os enfermeiros" de Coelho e Sequeira (2014), foi realizada a pesquisa com 448 enfermeiros, com faixa etária predominante entre 20 e 30 anos, a esses foram feitas perguntas sobre comunicação terapêutica na abordagem psiquiátrica. A partir das buscas de literaturas identificou-se que, até o momento, existem poucas publicações nacionais e internacionais acerca da comunicação terapêutica entre profissionais de enfermagem e pessoas com transtornos mentais, implicando no fato de não poder comparar os resultados desse estudo com outras pesquisas.

Todavia, através dos desfechos do artigo pode-se observar que a percepção quanto ao uso intencional e ao valor clínico autónomo da comunicação terapêutica não é unânime entre os profissionais. Sob a reflexão da literatura entende-se que a comunicação é um processo complexo, que envolve diversos fatores, na qual não será vivida da mesma forma, cada interação terá uma forma de comunicação diferente. Além disso, a comunicação entre profissional e paciente pode acabar sendo dificultada pelo que ela chama de "falsas crenças" ou "crenças limitantes", que podem acabar dificultando o entendimento do outro desde o início, sendo preciso diferenciar o que condiz com a realidade e não deixar influenciar por "mitos" (COELHO, 2015).

Outra divergência encontrada no artigo de Coelho e Sequeira (2014), entre os profissionais, foi quanto ao questionamento dos profissionais se os mesmos consideravam que toda comunicação utilizada pelo enfermeiro é terapêutica. Notou-se que à medida que os profissionais possuíam nível de formação mais elevado, a discordância com a frase tornava-se mais clara e evidente. Isso demanda atenção para a importância de discutir comunicação terapêutica durante o processo de formação profissional e das experiências obtidas ao longo dos anos de trabalho. 
Durante a graduação, o ensino tem algumas ressalvas no que diz respeito ao processo comunicativo. Essa ocorre pois a maioria dos cursos não dispõem de uma abordagem teórico- prática acerca do assunto, formando profissionais que não conseguem assistir os indivíduos holisticamente, necessitando que seja ofertada pelos gestores uma educação continuada, além da avaliação da conduta profissional frente ao paciente psiquiátrico, para que a partir da comunicação terapêutica se alcance a fragmentação dos bloqueios na relação enfermeiro- indivíduo (SILVA; BARROS, 2015).

\section{Comunicação terapêutica entre acadêmicos de enfermagem e pacientes com transtornos mentais}

Foram analisados três artigos a respeito da percepção dos acadêmicos de enfermagem, ambos referentes a uma vivência da disciplina de Saúde Mental numa instituição pública do Rio de Janeiro. Na perspectiva de unir os pontos de convergência entre os três artigos, é importante ressaltar que todos abordam a questão da comunicação a partir da sensação, percepção e compreensão dos estudantes de enfermagem.

É de suma importância analisar a percepção de acadêmicos sobre pessoas com transtornos mentais, visto que há um estigma que causa medo e dúvidas, e que impacta diretamente no processo comunicativo e na criação do vínculo profissional-paciente, provocando um distanciamento das experiências e do aprendizado (FERREIRA, 2018).

Os artigos de Azevedo, Araújo e Vidal (2015) e Azevedo et al (2017a e 2017b) abordam os comportamentos dos graduandos e a forma com que aprendem e percebem a comunicação através dos sentidos corporais. Ao longo do estudo, enfatiza-se a necessidade de um cuidado de enfermagem de qualidade, que é estabelecido à medida que o discente compreende o seu papel no curso da disciplina. É afirmado em vários momentos o quanto o estudante precisa se preparar para ser essencialmente humano, ao usar da empatia como 
ferramenta para estabelecer um relacionamento terapêutico no elo estudantepaciente e então, futuramente, profissional- paciente.

Os sentidos, explanados nesses estudos, são a forma de descrever de forma inovadora, mesmo que não única, a comunicação. A princípio, o coração é colocado na linha de frente como àquele que 'faz os demais sentidos sentirem', sendo representado pelo cuidado e pela já mencionada empatia que faz o estudante ir além do prescrito, agindo e reagindo em função da assistência efetiva ao paciente. Seguido pelo olfato, descrito como mais direto e importante para abrir margem à aprendizagem através de intervenções.

O paladar é sentido como íntimo e social, de atração ou repulsa, embora pouco descrito pelos estudantes. Reflete comportamentos, expressões e figuras de linguagem capazes de decifrar as inquietações e angústias dos transtornos mentais por atenção, cuidado e solidão vivenciada pelos pacientes. A escuta é colocada como primordial para a comunicação terapêutica pois carrega a sensibilidade do ouvir. Ainda nos sentidos, a visão é descrita como a matriz que toca através do olhar, e que juntamente com o tato imprime singularidades essenciais na interação.

Sendo assim, nota-se que os discentes compreendem que a comunicação terapêutica é vital para a promoção do bem estar biopsicossocial-espiritual e emocional do cliente de maneira a conduzi-lo às melhores formas de enfrentamento de suas condições (SILVA; BARROS, 2015). Porém, também entendem que essa comunicação não se restringe a linguagem verbal, mas considera todo e qualquer tipo de expressão.

\section{CONCLUSÃO}

Neste estudo, observou-se que tanto profissionais quanto estudantes percebem a comunicação terapêutica como ferramenta para efetivar relacionamentos terapêuticos, bem como auxiliar na oferta de cuidados em saúde e promover qualidade de vida aos pacientes com transtornos mentais. 
Além disso, os discentes reconhecem e destacam a relevância de considerartodos os sentidos do corpo humano (visão, olfato, paladar, audição e tato) como forma de comunicação.

Entretanto, isso somente é alcançado quando estudantes e profissionais de enfermagem estão capacitados para compreender a comunicação terapêutica e aplicá-la. Por isso, evidencia- se a importância do fortalecimento dos espaços educativos e de formação profissional para fornecer competências e habilidades na utilização da comunicação terapêutica.

A análise final dos artigos permitiu identificar a existência de poucas publicações nacionais e internacionais acerca da temática, implicando no fato de não poder comparar e melhor discutir os resultados com outras pesquisas. Portanto, espera-se que estudos como esse possam contribuir para a produção de novos artigos científicos e colabore para a reflexão da enfermagem sobre a comunicação como parte do processo de humanização do cuidar.

\section{REFERÊNCIAS BIBLIOGRÁFICAS}

ANDRADE, Kátia Cenira da Silva et al. Comunicação Terapêutica: instrumento básico do cuidado em crianças hospitalizadas. Rev Enferm Ufpe On Line, Recife, v. 9, n. 11, p.97849792, nov. 2015.

AZEVEDO, Albert Lengruber de; ARAÚJO, Sílvia Teresa Carvalho de; VIDAL, Veronica Lopes Louzada. Como o estudante de enfermagem percebe a comunicação com o paciente em saúde mental. Acta Paulista de Enfermagem, São Paulo, v. 28, n. 2, p.125-131, abr. 2015.

AZEVEDO, Albert Lengruber de et al. A comunicação do estudante de enfermagem na escuta de pacientes em hospital psiquiátrico. Escola Anna Nery, Rio de Janeiro, v. 21, n. 3, $2017 a$.

AZEVEDO, Albert Lengruber de et al. Comportamentos de graduandos de enfermagem na aprendizagem da comunicação em saúde mental. Rev. Enferm. UFPE On Line, Recife, v. 11 , n. 10, p.3878-3884, out. 2017b.

BRANCO, Paulo Coelho Castelo; CIRINO, Sérgio Dias. Reflexões sobre a consciência na fenomenologia e na abordagem centrada na pessoa. Gerais: Revista Interinstitucional de Psicologia, Minas Gerais, v. 9, n. 2, p.241-258, jul.-dez. 2016. CENCI, Mariana. 0 cuidado na saúde mental: trabalho do enfermeiro no Centro de Atenção Psicossocial. 67 f. TCC (Graduação) - Curso de Enfermagem, Centro Universitário Univates, Lajeado, 2015.

COELHO, Maria Teresa Vieira. Comunicação terapêutica em Enfermagem: utilização pelos enfermeiros. 276 f. Tese (Doutorado) - Curso de Enfermagem, Instituto de Ciências 
Biomédicas Abel Salazar, Universidade de Porto, Porto, 2015.

COELHO, Maria Teresa Vieira; SEQUEIRA, Carlos. Comunicação terapêutica em enfermagem: Como a caracterizam os enfermeiros. Revista Portuguesa de Enfermagem de Saúde Mental, Porto, n. 11, p.31-38, jun. 2014.

FERREIRA, Marcela dos Santos. Influência do ensino de saúde mental na modificação de atitudes estigmatizantes de alunos técnicos de enfermagem. $141 \mathrm{f}$. Dissertação (Mestrado) - Curso de Educação Profissional em Saúde, Escola Politécnica de Saúde Joaquim Venâncio, Fundação Oswaldo Cruz, Rio de Janeiro, 2018.

GALVÃO, Taís Freire; PANSANI, Thais de Souza Andrade; HARRAD, David. Principais itens para relatar Revisões sistemáticas e Meta-análises: A recomendação PRISMA. Instituto Evandro Chagas. Epidemiol. Serv. Saúde, Brasília, v. 24, n. 2, p.335-342, abr.jun. 2015.

JALLES, Marina Paranhos; SANTOS, Viviane Silva Januário dos; REINALDO, Amanda Márcia dos Santos. Análise da produção científica sobre comunicação terapêutica no campo da saúde, saúde mental e álcool e outras drogas. Rev. Med., São Paulo, v. 96, n. 4, p.232240, out.-dez. 2017.

PAIVA, Raquel de. Espiritualidade, Religiosidade e Subjetividade no Contexto do Sofrimento Psíquico Grave. Ecos-estudos Contemporâneos da Subjetividade, Niterói, v. 8, n. 2, p.278- 290, 2018.

PEREIRA, Bárbara Caroliny et al. Comunicação interpessoal e sua implicação na enfermagem.

Cultura de los cuidados, p. 230-238, jul. 2019.

RADKE, Mariane Brusque; CECCIM, Ricardo Burg. Educação em saúde mental: ação da reforma psiquiátrica no Brasil. Saúde em Redes, Porto Alegre, v. 4, n. 2, p.19-36, 2018.

SALVIANO, Márcia Eller Miranda et al. Epistemologia do cuidado de enfermagem: uma reflexão sobre suas bases. Rev. Bras. Enferm. [Internet], v. 69, n. 6, p.1240-1245, dez. 2016.

SILVA, Rodrigo Cardoso da; BARROS, Cleiciane Vieira de Lima. Comunicação terapêutica relacionada ao cuidado humanizado e a segurança do paciente em unidade hospitalar. Saúde \& Ciência em Ação: Revista Acadêmica do Instituto de Ciências da Saúde, São Paulo, v. 1, n. 01, p.13-25, jul. 2015. 